\title{
Évaluation des technologies éducatives émergentes \\ Les aléas de la conception, un facteur d'instabilité pour la définition des problématiques de recherche?
}

\section{Evaluating computer-based learning environments: hazards of the design process, a source of instability when establishing research questions?}

\section{Evaluación de entornos digitales: problemas del proceso de diseño, una fuente de inestabilidad al establecer preguntas de investigación?}

https://doi.org/10.52358/mm.vi8.225

Matthieu Cisel, enseignant-chercheur

CY Cergy Paris Université, France

matthieu.cisel@cyu.fr

RÉSUMÉ

En France, on demande de manière croissante aux laboratoires d'accompagner les projets de conception de technologies éducatives, sans nécessairement les mettre en position d'influer significativement sur ledit processus de conception. Pour pouvoir produire des résultats scientifiques, les chercheurs sont mis face à la nécessité de formuler leurs problématiques de sorte que les inévitables aléas de projets de conception inscrits dans la durée n'affectent pas de manière conséquente la productivité de leur travail. Dans cette contribution fondée sur un cas d'étude, le Carnet Numérique de l'Élève-Chercheur, nous revenons sur les trajectoires de plusieurs problématiques explorées dans le cadre de sa conception. Là où certaines sont imperméables aux difficultés du projet, d'autres doivent disparaître ou évoluer du fait de problèmes techniques rencontrés par le prototype, voire apparaissent en cours de conception, 
quand apparaît l'opportunité de produire des résultats originaux. Nous détaillons, sous la forme d'un retour d'expérience, les raisons sous-tendant ces choix.

Mots-clés : conception, problématisation, EIAH, évaluation

\section{ABSTRACT}

In France, research laboratories are increasingly asked to join educational technology design projects, in which they are not in a position in which they can influence design choices significantly. To produce scientific results, researchers are faced with the need to formulate their research questions so that the inevitable hazards of long-term design projects do not significantly affect the productivity of their work. In this contribution, based on a case study, the Student-Researcher Digital Notebook, we highlight the trajectories of different research questions. Some remained unchanged, while the others needed to disappear or evolve due to technical issues with the prototype. A question appeared during the design process when the opportunity arises to produce original results. We detail the reasons behind these choices.

Keywords: design, research question, computer-based learning environment, assessment

\section{RESUMEN}

En Francia, regularmente se solicita a los laboratorios que apoyen proyectos de diseño de tecnologías educativas, sin que necesariamente los coloquen en condiciones de influir fuertemente en dicho proceso de diseño. Para poder producir resultados científicos, los investigadores se enfrentan a la necesidad de formular sus problemas de tal manera que los inevitables problemas de los proyectos de diseño a largo plazo no afecten significativamente la productividad de su trabajo. En esta contribución basada en un estudio de caso, el Cuaderno Digital del Alumno-Investigador, volvemos a varias trayectorias de problemas. Unos no cambiaron, pero otros tuvieron que desaparecer o evolucionar ligeramente debido a problemas técnicos con el prototipo. Un problema aparezco durante el diseño, cuando surge la oportunidad de producir resultados originales. Este último se abandono por razones científicas. Detallamos las razones detrás de estas elecciones.

Palabras clave: diseño, utilidad, pregunta de investigación, entorno digital, evaluación

\section{Introduction}

\section{Différentes approches de la recherche en technologies éducatives}

Dans le champ des technologies éducatives, la recherche est de plus en plus encouragée, via les financements publics, à participer à des projets débouchant sur des applications directement utilisables en classe. C'est notamment le cas des projets eFRAN (pour espaces de Formation, de Recherche et d'Animation Numérique) financés par la Caisse des dépôts et consignations (CDC) sur la période 
2016-2019. À travers les eFRAN, l'État français a encouragé la conception d'Environnements Informatiques pour l'Apprentissage Humain (EIAH) au sein de consortiums incluant systématiquement des laboratoires de recherche, sans que ceux-ci ne soient en position de rédiger le cahier des charges des applications. Lorsque les chercheurs ne sont ni en position de peser sensiblement sur les orientations technologiques de ces projets ni en position d'exercer un contrôle important sur le calendrier de développement, se pose le problème de la marge de manœuvre dont ils disposent dans la définition de leurs problématiques et de la sensibilité de leurs résultats aux aléas des projets de conception.

Cette considération nous a amené, sur la base d'une recherche de trois ans au service du développement du Carnet Numérique de l'Élève-Chercheur (CNEC) dans le consortium eFRAN Les Savanturiers du Numérique, à nous poser les questions suivantes, en tant que praticien: Dans quelle mesure les problématiques qui structurent l'activité des laboratoires impliqués dans des projets de conception d'EIAH, en position de recherche d'accompagnement, peuvent-elles être influencées par les aléas des projets? Comment formuler les problématiques traitées de telle sorte à ce qu'elles soient peu sensibles à ces aléas?

Cette contribution, qui prend la forme d'un retour d'expérience, vise avant tout à alimenter le débat sur les évolutions actuelles des politiques de financement des travaux scientifiques consacrés aux technologies éducatives. En nous attardant sur un cas d'étude, le CNEC, nous voulons illustrer comment la recherche peut être mise en difficulté lorsqu'elle a pour mission de produire des résultats sur des objets mouvants, dont elle ne maîtrise que marginalement les évolutions. Nous cherchons également à montrer comment l'on peut tenter de s'adapter aux aléas d'un projet pour transformer des obstacles en opportunités de s'engager sur des voies de recherche fertiles. Les Savanturiers du Numérique, projet eFRAN développé de 2016 à 2019, constitue du fait de sa complexité un cadre intéressant pour mener une telle réflexion. Revenons sur le contexte dans lequel s'est déroulée cette recherche, pour ensuite diviser nos axes de recherche en deux grandes catégories : ceux qui sont restés stables d'une part et ceux qui ont évolué au fil du temps d'autre part.

\section{Contexte de la recherche et méthodes mises en œuvre}

Le consortium Les Savanturiers du Numérique, réuni en 2016 pour une durée de trois ans, avait vocation à instrumenter les projets Savanturiers, un programme fondé en 2013 par une ancienne professeure des écoles (Royer, 2017; Carosin et Demeuse, 2018). Celui-ci vise à développer des projets de recherche miniatures, principalement à l'école primaire et au collège, et encadrés par des mentors généralement issus du milieu universitaire (Pirone, 2018). II s'agit d'initier les élèves aux démarches d'investigation (Coquidé, Fortin et Rumelhard, 2009), en leur faisant jouer une part active aux différentes étapes de la démarche, de la formulation de la question de recherche à l'interprétation des résultats. Le programme s'inscrit explicitement dans une logique de renouvellement des approches pédagogiques dans l'enseignement des sciences, mettant l'accent sur la dimension méthodologique de l'activité scientifique.

En tant que porteur du projet, Les Savanturiers disposent d'une position de choix pour peser sur le processus de conception, mais dans la mesure où le développement informatique est assuré par une entreprise, Tralalère ${ }^{1}$, c'est en définitive cette dernière qui contrôle les budgets de développement. Cette position est d'autant plus légitime que cet industriel est propriétaire du code et que la conception du CNEC s'inscrit dans sa stratégie propre. Les Académies de Paris et de Créteil facilitent l'accès au terrain et

\footnotetext{
${ }^{1}$ Le site du CNEC est disponible à cette adresse : https://www.cnec.fr/accueil.
} 
effectuent des retours utilisateurs. Enfin, le laboratoire EDA est responsable de la conduite d'une recherche, dont les axes n'étaient pas pleinement déterminés au moment du dépôt du projet. Seul le cadre théorique de la théorie de l'activité d'Engeström (1987) avait été fixé dès les premières étapes de la recherche.

Le CNEC est constitué de sept modules interconnectés (Cisel, Barbier et Baron, 2019). Les fonctionnalités associées à ces modules sont d'origines variées. Les fonctionnalités d'étayage portées par le module dit du Brouillon de recherche sont par exemple issues d'une proposition du laboratoire EDA, faisant suite à une revue de la littérature sur les technologies dans l'enseignement des sciences (Cisel et Baron, 2019a). D'autres modules, comme le Générateur d'idées, sont davantage issus des propositions de l'entreprise. En définitive, le CNEC partage de nombreuses similitudes avec le Knowledge Forum (Scardamalia et Bereiter, 2003, 2006; Laferrière et Lamon, 2010), le LabNBook (Bonnat, Marzin et d'Ham, 2019) ou le WISE (Slotta et Linn, 2009), deux environnements utilisés dans l'enseignement scientifique fondé sur l'investigation et qui ont fait l'objet d'une littérature abondante. II s'inscrit dans la lignée de nombreux artefacts visant à instrumenter ce que l'on qualifie d'enseignements scientifiques fondés sur l'investigation (Wu et Hsieh, 2006; Linn, 2000, 2013). La multiplicité des modules du CNEC ouvre la voie à des questions de recherche variées, à condition que l'artefact soit effectivement utilisable en classe. Dans la partie qui suit, nous déclinons chacun des sous-axes liés aux utilisations en classe des technologies éducatives.

En termes de méthodologie, nous avions la possibilité d'observer des projets instrumentés ou non par le CNEC. Au cours des trois années qu'a duré le consortium, dix projets Savanturiers ont été suivis de manière longitudinale, à raison de cinq à quinze séances par projet (Cisel, Barbier et Baron, 2019). II s'agissait de collèges et d'écoles primaires situées dans les Académies de Paris et de Créteil. Les observations pouvaient être complétées par des entretiens individuels et collectifs, des fonds pouvant être attribués aux enseignants pour les dédommager de leur temps. Enfin, nous avons assisté à l'essentiel des réunions liées au processus de conception. Nous avons observé le déroulé des réunions mensuelles, rassemblant généralement l'industriel, les Savanturiers et le laboratoire, et, six fois par an, les enseignants - nous parlons alors de comité de conception, ou CoCon. La participation au processus de conception et l'accès facilité aux classes ouvraient la possibilité d'explorer des pistes de recherche variées. Nous commencerons par présenter les axes qui sont restés stables au fil des trois ans.

\section{Définir des axes de recherche stables au cours du projet}

Les questions de recherches investiguées au sein du laboratoire EDA ont été divisées en trois axes. Le premier axe est centré autour du programme Savanturiers et s'inscrit dans la lignée de travaux préliminaires sur le sujet (Pirone, 2018). Nous nous sommes intéressé aux pratiques enseignantes développées en amont de l'introduction de l'artefact et aux tensions qui traversent le programme Savanturiers. Cette approche nous semble intéressante pour deux raisons. En premier lieu, il est recommandé dans le cadre d'un projet de conception d'établir un état de besoin sur la base d'observations de classe. II s'agit d'identifier l'intérêt d'une instrumentation numérique. En second lieu, les problématiques relatives au fonctionnement des projets Savanturiers sont insensibles aux aléas du calendrier de conception.

Le second axe correspond à l'étude du processus de conception. L'avantage de cet axe réside dans le fait que les difficultés et les aléas du processus de conception peuvent, dans cette approche, devenir un objet d'étude à part entière. L'analyse d'une matrice de décision utilisée pour réaliser des arbitrages en début 
de projet fait partie des travaux réalisés dans le cadre de cet axe (Cisel et al., 2017). Cet outil utilisé dans les premiers mois du projet était fondé sur des scores attribués à chaque acteur, pour notamment permettre aux enseignants de peser dans les orientations du projet. Nous avons montré mathématiquement que, bien que la pondération des scores leur soit favorable, le poids effectif des praticiens sur les décisions prises est négligeable. En suivant cette approche, les difficultés liées à la collaboration alimentaient nos travaux plus qu'elles ne les freinaient.

En guise de troisième axe, nous avons travaillé sur les utilisations de l'artefact en classe; les chercheurs impliqués dans le consortium ayant été associés en grande partie pour leur expérience dans le domaine de l'étude des technologies éducatives. Ce dernier axe est quant à lui relativement sensible aux aléas du processus de conception, car les problèmes techniques et ergonomiques interfèrent avec la collecte des données. Nous avons par exemple travaillé sur l'instrumentation de la rédaction incrémentale des idées (Vardi, 2012). Lorsque l'on s'inscrit dans cette logique, l'enseignant invite l'élève à reformuler son idée jusqu'à ce que sa formulation soit jugée satisfaisante, ce travail exigeant souvent que le texte fasse plusieurs allers-retours avant d'être validé définitivement. La scolarisation d'une telle technologie soulève notamment la question de la réorganisation de l'activité enseignante autour de l'artefact. Pour ne pas subir les aléas de la conception, nous avons en particulier fait le choix des méthodes qualitatives, plébiscitées dans la littérature sur les méthodologies d'évaluation des EIAH (Jamet, 2006). Tous les sujets ne se prêtent pas nécessairement à ce type d'approche; nous détaillons dans la section qui suit les différents axes qui ont évolué au fil des aléas de la conception.

\section{Des questions de recherche sensibles aux aléas de la conception}

Afin d'illustrer la diversité des configurations eu égard à l'évolution de nos problématiques, nous proposons de traiter trois questions de recherche, correspondant à autant de modules du CNEC. Nous commencerons par une question sur l'utilisation des étayages du Brouillon de recherche, qui a dû être transformée du fait des aléas du projet. La deuxième section sera consacrée aux tableaux de bord, thématique apparue en cours de projet et qui servira à illustrer notre volonté de saisir les opportunités qu'offraient des évolutions inattendues de la conception. Enfin, nous conclurons par les questions que nous avons renoncé à traiter, car elles nécessitaient un investissement jugé coûteux. L'une des principales difficultés dans ce type de projet consiste à formuler les questions de sorte à rendre les problématiques opérantes sur le plan scientifique et à ne pas être cantonné à un rôle de traitement de questions d'ergonomie et de conception pédagogique. Toutes les questions qui suivent sont ainsi pensées pour remplir cette condition, mais les aléas de la conception complexifient ce travail de délimitation.

\section{Une reformulation des problématiques relatives aux étayages}

Le module Brouillon de recherche a permis d'aborder un certain nombre de questions relatives aux étayages en enseignement des sciences. L'objectif du laboratoire était de faire réifier un certain nombre de propositions au sein du CNEC et, ce faisant, de mener des recherches sur l'efficacité des étayages. L'utilisation des étayages numériques dans le champ de la conception expérimentale constitue un domaine de recherche d'une actualité toujours vivace (Edelson, Gordin et Pea, 1999; Quintana, Zhang et Krajcik, 2005), y compris en France (Bonnat, 2017). Nous pensions à l'origine que le développement serait suffisamment rapide pour permettre une expérimentation randomisée avec prétest et post-test. Une telle 
approche aurait permis d'évaluer l'utilité d'une telle fonctionnalité sur la base des productions des élèves, dans la continuité des recherches doctorales de Bonnat (2017) et Saavedra (2015) sur le LabNBook. La question initiale pouvait être formulée en ces termes: Dans quelle mesure les étayages proposés par le CNEC contribuent-ils à l'amélioration des productions écrites des élèves aux étapes de formulation de la question de recherche, de l'éventuelle hypothèse et du protocole?

Les protocoles de type pré-test/post-test visent à mesurer une performance des élèves avant l'intervention impliquant la pratique pédagogique instrumentée et la performance après cette même intervention. On met généralement en place un groupe témoin d'élèves qui n'utilise pas la technologie et un groupe qui l'utilise, pour comparer ensuite les performances des deux groupes. Ce type d'approche a gagné en influence dans les discours avec la promotion, notamment institutionnelle, de l'evidence-based education (Slavin, 2002), que nous pourrions traduire par « instruction guidée par les résultats de recherche ». Si nos questions prêtaient à la mise en place de tels protocoles, les problèmes techniques se sont accumulés - les productions des élèves, par exemple, disparaissaient aléatoirement; nous détaillons dans une publication les problèmes rencontrés, qui incluent également les questions de logistique pour la constitution des groupes expérimentaux (Cisel, 2020). Enfin, l'ergonomie du module a engendré des obstacles divers, avec notamment des difficultés de lisibilité pour les élèves. Les conditions n'ont en définitive pas été réunies pour mener une expérimentation randomisée et l'accumulation des problèmes a fait obstacle à l'étude de l'utilité des étayages comme envisagé initialement. En d'autres termes, nous avons fait face aux difficultés associées à la mise en place de méthodes expérimentales en éducation, fréquemment évoquées dans la littérature scientifique (Baron et Bruillard, 2007; Biesta, 2010), d'autant que la généricité des résultats produits posait problème.

Une expérimentation randomisée a davantage de sens lorsqu'un EIAH est stabilisé techniquement et sur le point d'être industrialisé - il peut alors servir à évaluer la pertinence d'un tel passage à l'échelle, ou lorsqu'une technologie a été scolarisée depuis plusieurs années et que l'on souhaite appréhender son utilité en conditions écologiques. Dans le cadre du projet Les Savanturiers du Numérique, nous ne nous situions ni dans l'une ni dans l'autre de ces configurations. Nous avions sous-estimé le temps que prendrait la mise au point d'un prototype stable, ce qui a délégitimé l'approche envisagée initialement et nous a réorienté vers des approches plus qualitatives (Cisel, 2020).

Pour ne pas perdre les bénéfices de la réflexion engagée sur la thématique et dans la mesure où, malgré des difficultés techniques, un module portant des étayages avait été développé à notre demande, nous avons préféré une reformulation des questions de recherche associées plutôt qu'un abandon pur et simple de l'axe de recherche. Ce travail de reformulation a conduit à traiter, à partir de la fin du projet, les problématiques suivantes : De quelle manière les praticiens se sont-ils approprié les étayages visant à structurer les démarches d'investigation? Quels sont les obstacles à l'utilisation de tels étayages pour les pratiques enseignantes? Cette approche a permis de ne pas perdre totalement les bénéfices de la réflexion menée en premier abord. Néanmoins, le coût associé à la formulation d'une question sensible aux aléas de la conception a été conséquent, ne serait-ce que du fait de l'investissement réalisé dans une étude de faisabilité des protocoles associés. L'objet de recherche a été conservé, mais l'angle a été modifié pour prendre en compte les aléas du projet. 


\section{Un axe de recherche apparaissant au fil du projet : les tableaux de bord}

Nous en arrivons maintenant à un autre cas de figure : les aléas du projet ont inspiré la mise en place d'un axe de recherche nouveau. Dès la fin de la première année du projet, émerge au sein du consortium l'idée de développer un tableau de bord (TDB) à destination de l'enseignant et dont la vocation est d'instrumenter le suivi des actions des élèves. Par exemple, le TDB permettrait de visualiser quand un groupe ou un élève s'investissait quantitativement moins que les autres dans la rédaction d'idées, ou le nombre d'allers-retours effectués avant d'arriver à une production jugée satisfaisante. Cette fonctionnalité, portée notamment par les Savanturiers, s'accompagne d'une proposition d'usage: l'utilisation de TDB pour étayer des évaluations des compétences scolaires (Cisel et Baron, 2019b). Le consortium a mandaté un consultant pour suggérer d'associer des indicateurs à divers éléments du socle commun évalués au collège.

Cette proposition de TDB, qui s'inscrit dans la continuité de nombreux travaux anglo-saxons (Verbert et al., 2014) et francophones (Carillo et al., 2018) sur la question, nous a semblé représenter l'occasion d'apporter un regard critique sur un sujet relativement nouveau. Ces derniers sont en effet enjoints à utiliser des applications numériques d'une part - certains transmettent des informations sur les actions des élèves - et à effectuer d'autre part une transition d'une évaluation par notes à une évaluation par compétences, considération qui nous a amené à étudier la problématique suivante: Quelles sont les contradictions potentiellement introduites, lors de la réalisation des évaluations des compétences scolaires, par l'utilisation de TDB fondés sur des indicateurs d'activité des élèves?

La méthodologie a été choisie en prenant en compte le fait que le TDB risquait de ne pas être développé : nous avons choisi de travailler à partir de maquettes - les propositions d'indicateurs n'ont en effet pas été réifiées dans le code faute de budget - et avons suivi une méthode qualitative fondée sur des entretiens. Cette piste de recherche, fertile au demeurant (Cisel et Baron, 2019b), est apparue d'autant plus pertinente que progresse la scolarisation des tableaux de bord issus d'applications éducatives. Contrairement aux questions précédentes, qui ont été formulées dès les premières étapes du projet de conception, celle-ci est apparue du fait d'évolutions inattendues du processus de conception. Néanmoins, cette approche implique de pouvoir distinguer les opportunités intéressantes de celles, plus risquées, qui peuvent déboucher sur des résultats difficiles à valoriser scientifiquement et donc à abandonner un axe de recherche.

\section{Renoncer à un axe de recherche : le cas du Générateur d'idées}

Le Générateur d'idées est une proposition défendue avant tout par l'entreprise, mais qui trouve sa légitimité dans les discours du programme Savanturiers. C'est un "mur d'idées », type d'application visant à mutualiser les idées d'une classe ou d'un groupe d'élèves. En tant que système de gestion d'idées (SGI), ou Idea Management System (IMS) dans la littérature anglo-saxonne (Sandstrom et Bjork, 2010), il s'apparente aux applications comme le Padlet, très utilisées par les praticiens, aussi bien à l'école primaire qu'au collège. De par son développement rapide, c'est le module qui offrait le plus d'opportunités de collecte de données; les questions de recherche centrées sur cet IMS ont néanmoins été rapidement abandonnées.

Pour le scientifique, la difficulté que posent les recherches sur un tel objet réside dans le fait que ce type d'application a fait l'objet de décennies de recherche, auxquelles appartiennent notamment celles sur le Knowledge Forum (Scardamalia et Bereiter, 2003, 2006, 2013). II nous est apparu coûteux d'identifier des pistes de recherche susceptibles de produire des résultats originaux. Le risque est élevé de produire des 
résultats triviaux, ou redondants avec des travaux déjà publiés. Alors même que c'est sur le Générateur d'idées que nous avions le plus de données - le module a été développé en premier - nous avons dû renoncer à développer une réflexion spécifique sur ces questions.

Enfin, une brève recherche bibliographique a suffi à identifier des résultats analogues à ceux que nous observions en classe (Fuchs, 2014), ce qui laisse supposer que l'intérêt scientifique qu'il y a à documenter toutes les difficultés observées en classe reste faible. Nos observations avaient tout au plus un intérêt du point de vue de la conception et ont été principalement rapportées aux programmeurs. La valeur ajoutée de notre position de chercheur était faible. Nous avons considéré que saisir toutes les opportunités que nous offrait le CNEC augmentait sensiblement le risque de dispersion et qu'il était préférable de se concentrer sur un nombre plus limité de problématiques, que nous traiterions de manière plus approfondie. Après avoir effectué une synthèse des trajectoires des différentes problématiques envisagées, nous conclurons cette contribution sur différentes perspectives de recherche.

\section{La posture délicate de la recherche d'accompagnement}

Dans ses travaux de référence sur la conception d'EIAH, Tchounikine (2002; 2011) décrit une « voie royale " du point de vue scientifique, où le chercheur pilote les développements en fonction de ses questions de recherche et de ses hypothèses. Néanmoins, cette voie suppose de disposer du temps et de la capacité à programmer, et les projets eFRAN ont préféré donner aux laboratoires un rôle d'accompagnement plus que de pilote. $\mathrm{Si}$, dans les années à venir, cette posture se conforte dans les projets de conception d'applications numériques, elle pourrait selon nous accroître les incompréhensions au sein de la communauté scientifique travaillant sur les EIAH. Nous avons pu constater les divergences de perspectives entre les chercheurs inscrits dans une logique de recherche d'accompagnement et ceux qui sont accoutumés à inféoder les orientations technologiques des artefacts qu'ils développent à leurs questions de recherche, qu'elles soient de nature didactique, informatique ou autre.

Pour que la recherche d'accompagnement n'apparaisse pas dans le champ des technologies éducatives comme une recherche par défaut, fruit de choix politiques que ne maîtrise pas la communauté scientifique, il nous semble essentiel de faire connaître davantage, au sein de la communauté EIAH, les contraintes auxquelles elle est confrontée. En particulier, il nous apparaît intéressant de rendre plus explicites les trajectoires des projets en termes de questionnement scientifique. Une telle démarche permettrait de fortifier la réflexion sur la manière de construire des problématiques suffisamment robustes pour résister aux aléas des processus de conception.

En guise de conclusion, soulignons qu'il serait sans doute pertinent d'élargir la réflexion sur la définition des questions de recherche initiée au sein du consortium Les Savanturiers du Numérique et notamment à l'ensemble des projets eFRAN où il a été question de développer ou d'améliorer un EIAH. Nous pourrions contraster les différentes situations pour appréhender comment les chercheurs impliqués ont adapté leurs problématiques aux évolutions des projets dans lesquels ils s'impliquaient. À partir des données collectées par les financeurs de ces recherches - la Caisse des dépôts dans le cas présent -, il est notamment possible de se pencher sur la manière dont les caractéristiques d'un projet - place du laboratoire, niveau d'avancement du processus de conception, etc. - influent sur la productivité scientifique. La CDC a recensé de manière aussi exhaustive que possible les publications issues des projets eFRAN sans avoir, à notre connaissance, cherché à relier productivité et contexte de développement des recherches. Or ce travail permettrait de mieux appréhender comment les modalités de financement de la recherche affectent 
la nature des résultats publiés et la productivité des laboratoires. Il convient en effet de s'interroger quant aux conditions nécessaires pour préserver une recherche scientifique de qualité malgré la participation des laboratoires dans des projets à la visée pratique assumée.

\section{Liste de références}

Baron, G.-L. et Bruillard, E. (2007). ICT, educational technology and educational instruments. Will what has worked work again elsewhere in the future? Education and Information Technologies, 12(2), 71-81. https://doi.org/10.1007/s10639-007-9033-9

Biesta, G. J. J. (2010). Why 'What Works' Still Won't Work: From Evidence-Based Education to Value-Based Education. Studies in Philosophy and Education, 29(5), 491-503. https://doi.org/10.1007/s11217-010-9191-x

Bonnat, C. (2017). Etayage de l'activité de conception expérimentale par un EIAH pour apprendre la notion de métabolisme cellulaire en terminale scientifique. Manuscrit de thèse non publié. Université Grenoble Alpes, France.

Bonnat, C, Marzin, J. P et d'Ham, C. (2019). Modélisation didactique pour la conception d'étayages dans un EIAH : Exemple d'une activité de conception expérimentale en biologie. STICEF, 25. https://hal.archives-ouvertes.fr/hal-02015388

Cisel, M., Beauné, A, Bernard, F., Voulgre, E. et Baron, G. (2017). Analyse d'un outil de décision mobilisé dans la conception d'un EIAH. Dans Actes de la $8^{e}$ conférence sur les Environnements Informatiques pour l'Apprentissage Humain, 382-385.

Cisel, M. et Baron, G. (2019a). Vers des intelligences artificielles pour l'enseignement du raisonnement scientifique. Dans Séminaire Inter-Laboratoires sur l'Education Scientifique et Technologique. Patras, 3-5 avril 2019.

Cisel, M. et Baron, G.-L. (2019b). Utilisation de tableaux de bord numériques pour l'évaluation des compétences scolaires : Une étude de cas. Questions Vives. Recherches en éducation, 31. https://doi.org/10.4000/questionsvives.3883

Cisel, M., Barbier, C. et Baron, G.-L. (2019). Rapport scientifique de synthèse de la recherche Cahier numérique de l'élève chercheur (CNEC). Université Paris Descartes (Paris 5). https://hal.archives-ouvertes.fr/hal-02278348

Cisel, M. (2020). Évaluer l'utilité d'un EIAH : difficultés rencontrées lors d'une expérience randomisée, STICEF, 27, 1. https://hal.archives-ouvertes.fr/hal-03245831

Coquidé, M. Fortin, C. et Rumelhard, G. (2009). L'investigation : fondements et démarches, intérêts et limites. ASTER. 49, 51-78. https://doi.org/10.4267/2042/31129

Edelson, D. C., Gordin, D. N. et Pea, R. D. (1999). Addressing the Challenges of Inquiry-Based Learning Through Technology and Curriculum Design. Journal of the Learning Sciences, 8(3-4), 391-450. https://doi.org/10.1080/10508406.1999.9672075

Engeström, Y. (1987). Learning by expanding: An activity-theoretical approach to developmental research. Helsinki, Finland: Orienta-Kosultit Oy.

Fuchs, B. (2014). The writing is on the wall: using Padlet for whole-class engagement. LOEX Quarterly, 40(4), 7. https://commons.emich.edu/loexquarterly/vol40/iss4/4/

Jamet, E. (2006). Une présentation des principales méthodes d'évaluation des EIAH en psychologie cognitive. STICEF, 13. https://hal.archives-ouvertes.fr/hal-00696350

Laferrière, T. et Lamon, M. (2010). Knowledge Building/Knowledge Forum®: The Transformation of Classroom Discourse. Dans M. S. Khine et I. M. Saleh (Eds.), New Science of Learning (pp. 485-501). New York, NY: Springer.

Linn, M. C. (2000). Designing the Knowledge Integration Environment. International Journal of Science Education, 22(8), 781-796. https://doi.org/10.1080/095006900412275

Linn, M. C. (2013). Internet Environments for Science Education. Abingdon-on-Thames, UK: Routledge.

Pirone, F. (2018) « Les Savanturiers de la sociologie ». De la recherche en « éducation par la recherche ». Diversité. 192. 
Quintana, C., Zhang, M. et Krajcik, J. (2005). A Framework for Supporting Metacognitive Aspects of Online Inquiry Through Software-Based Scaffolding. Educational Psychologist, 40(4), 235-244.

https://doi.org/10.1207/s15326985ep4004 5

Royer, M. (2017). Les Savanturiers : le chemin de l'investigation scientifique. Les Cahiers Pédagogiques. http://www.cahiers-pedagogiques.com/Les-Savanturiers-le-chemin-de-l-investigation-scientifique

Saavedra, R. (2015). Etayer le travail des élèves avec la plateforme LabBook pour donner davantage de sens aux activités expérimentales réalisées par des élèves de première $S$. Manuscrit de thèse non publié, Université Grenoble-Alpes, France.

Sandstrom, C. et Bjork, J. (2010). Idea management systems for a changing innovation landscape. International Journal of Product Development, 11(3-4), 310-324. https://www.inderscience.com/info/inarticle.php?artid=33964

Scardamalia, M. et Bereiter, C. (2003). Knowledge building environments: Extending the limits of the possible in education and knowledge work. Encyclopedia of Distributed Learning, 269-272.

Scardamalia, M. et Bereiter, C. (2006). Knowledge Building: Theory, Pedagogy, and Technology. In K. Sawyer (Ed.), Cambridge Handbook of the Learning Sciences (p. 97-118). New York: Cambridge University Press.

Scardamalia, M. et Bereiter, C. (2013). An Architecture for Collaborative Knowledge Building. In Computer-Based Learning Environments and Problem Solving. Springer Science et Business Media.

Slavin, R. E. (2002). Evidence-Based Education Policies: Transforming Educational Practice and Research. Educational Researcher, 31(7), 15-21. https://doi.org/10.3102/0013189X031007015

Slotta, J. D. et Linn, M. C. (2009). WISE Science: Web-Based Inquiry in the Classroom. New York, NY, USA: Teachers College Press.

Tchounikine, P. (2002). Quelques éléments sur la conception et l'ingénierie des EIAH. Actes des $2^{e}$ assises nationales du GdR I3 - Groupe de Recherche Information Interaction Intelligence, décembre 2002. https://telearn.archives-ouvertes.fr/hal-00190110

Tchounikine, P. (2011). Computer Science and Educational Software design - A Resource for Multidisciplinary Work in Technology Enhanced Learning. Berlin, Allemagne : Springer.

Vardi, I. (2012). The impact of iterative writing and feedback on the characteristics of tertiary students' written texts. Teaching in higher education, 17(2), 167-179. https://doi.org/10.1080/13562517.2011.611865

Verbert, K., Govaerts, S., Duval, E., Santos, J., Van Assche, F., Parra, G. et al. (2014). Learning dashboards: An overview and future research opportunities. Personal and Ubiquitous Computing, 18(6), 1499-1514. https://doi.org/10.1007/s00779-013-0751-2

Wu, H.-K. et Hsieh, C.-E. (2006). Developing Sixth Graders' Inquiry Skills to Construct Explanations in Inquiry-based Learning Environments. International Journal of Science Education, 28(11), 1289-1313. https://doi.org/10.1080/09500690600621035 